GESNERUS

Jahrgang/Vol.44 1987 Heft/Fasc. 3/4

Vierteljahrsschrift für Geschichte

der Medizin und der Naturwissenschaften

Revue trimestrielle d'histoire

de la médecine et des sciences naturelles

Redaktion/Rédaction:

Carl Haffter, Basel

Verlag/Editions Sauerländer, Aarau

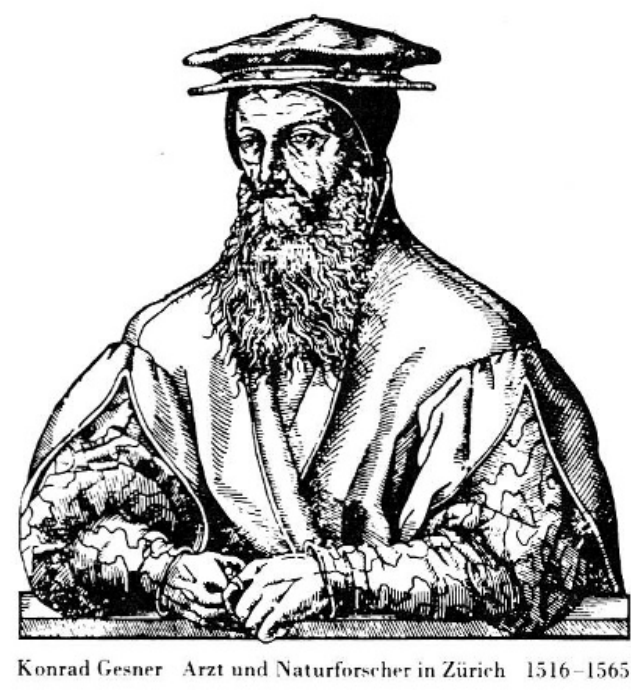

\title{
Die opopira magna, ein pharmazeutisches Präparat aus dem Antidotarius magnus
}

Von Willem F. Daems und François Ledermann

Von den zahlreichen Initialen, die den 1959 von Alfons Lutz wiederentdeckten «Liber Antidotarius magnus» der Universitätsbibliothek Basel schmükken $^{1}$, sind einige in den letzten Jahren beschrieben worden ${ }^{2}$. Einem weiteren Bilde, das die Heiligen Kosmas und Damian zeigt, hat man bis vor kurzem keine große Aufmerksamkeit geschenkt, vielleicht weil es eine mindere künstlerische Qualität aufweist ${ }^{3}$. Die Schutzheiligen der Ärzte, der Chirurgen und der Apotheker, beide mit einem Palmzweig und einem langen antiken Gewand, bilden ein O: es handelt sich um den Beginn der Beschreibung eines pharmazeutischen Präparates, der großen Opopira, «Opopira magna».

Die verschiedenen Wunderberichte von Kosmas und Damian ${ }^{4}$ - gleichgültig ob es sich um Wunder zu Lebzeiten oder Miracula post mortem handelt - enthalten nur wenige pharmazeutische Produkte, denn die Heiligen, obwohl sie eindeutig dem Ärztestand zugeschrieben wurden, behandelten ihre Patienten meistens, indem sie zu einer Art mit ihrer religiösen Macht innig verbundenen Psychotherapie Zuflucht nahmen; die von ihnen praktizierte Inkubation, Erbe der antiken Medizin und Therapie, bringt das beste Beispiel davon ${ }^{5}$. Da aber der Ruf der beiden Heiligen in Westeuropa zu Beginn des Mittelalters als Folge einer Devotionswelle 


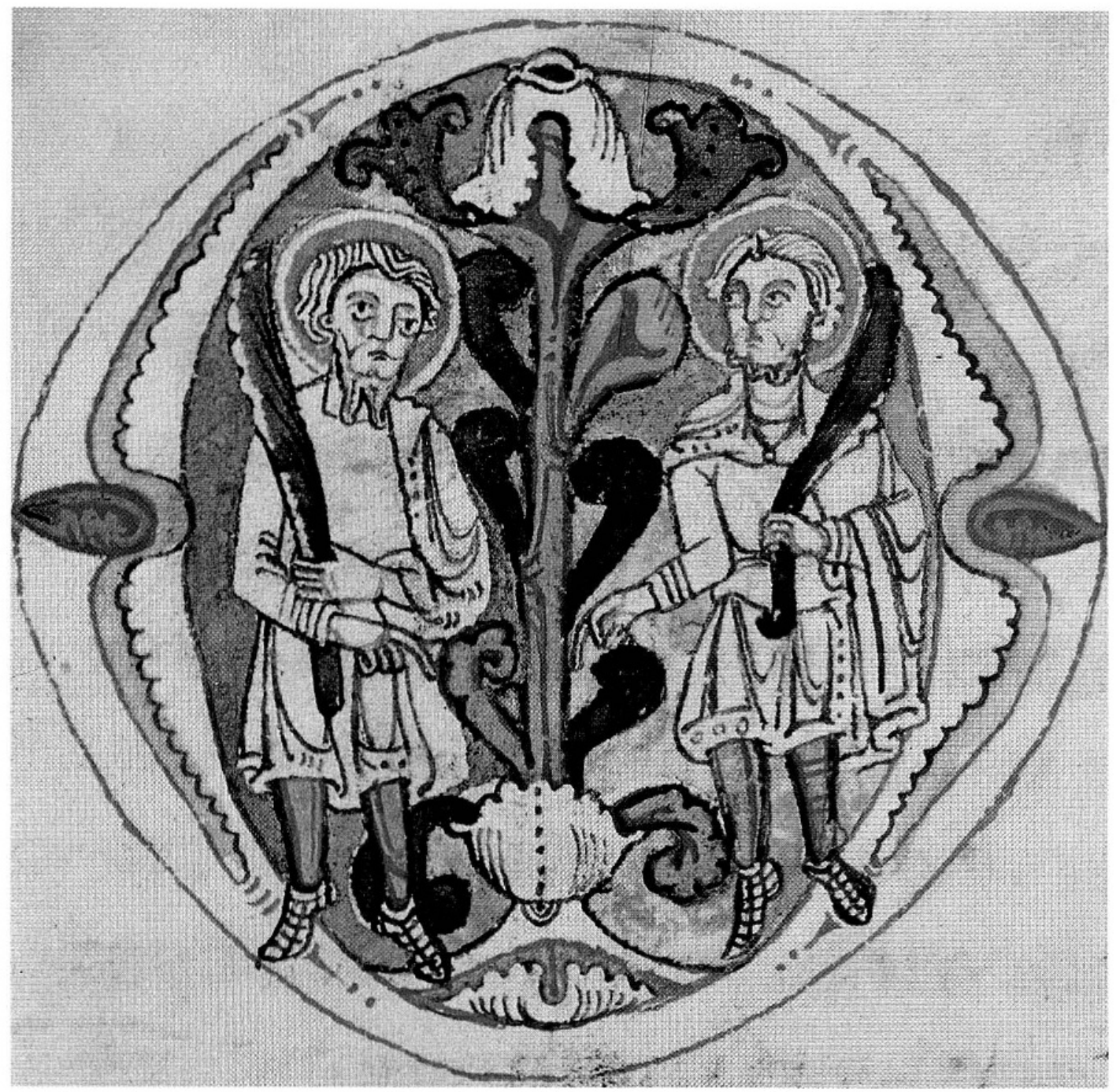

Basel, Universitätsbibliothek, Ms. D III 14, f.84r

Kosmas und Damian in O-Initiale der 'Opopira'-Vorschrift

wuchs ${ }^{6}$, wurden einige Medikamente Kosmas und Damian zugeschrieben, wahrscheinlich, damit ihre Wirkung aus der thaumaturgischen Kraft, die man den beiden Heiligen zuschrieb, referenzbedingten Nutzen ziehen konnte ${ }^{7}$; unter diesen Arzneimitteln erscheint die Opopira, welche zuerst in der pharmazeutischen Literatur salernitanischen Ursprungs hervortritt. Es scheint, daß Constantinus Africanus der erste war, welcher diese Zusammensetzung erwähnt, ohne sie jedoch den Heiligen Kosmas und Damian zuzuschreiben. Er vermittelt sogar den Eindruck, er sei selber der Erfinder des Präparates, denn er schreibt: «Pro gutta, podagra, paralysi, da pilulas, \& benedicta, vel opopira nostrum.» ${ }^{8}$ 
So taucht die Opopira ganz natürlich sowohl im Antidotarius magnus auf, einem Werk dessen Beziehungen zu Salerno offenkundig sind ${ }^{9}$, als auch im Antidotarium Nicolai, das, wie Alfons Lutz zeigen konnte, eine zusammengefaßte, mehr zugängliche und oft halb-offizielle Neufassung des großen Salernitanischen Antidotars darstellt ${ }^{10}$. Die Opopira wird noch von Arnaldus Villanova erwähnt, der nicht unterläßt zu erwähnen, daß das Präparat von Kosmas und Damian erfunden wurde: «Opopyra quam sanctissimi viri Cosmus \& Damianus composuerunt.» ${ }^{11}$ Dann scheint sie aus den pharmazeutischen Formularien zu verschwinden, erscheint jedenfalls in den ersten Stadtpharmakopöen von Augsburg und Nürnberg nicht mehr ${ }^{12}$.

Der hier übersetzte und kommentierte Text der Opopira, aus der aus Einsiedeln stammenden Handschrift der Universitätsbibliothek Basel ${ }^{13}$, ist anderen Fassungen des «Großen Antidotars» ähnlich; insbesondere die drei Handschriften der Bibliothèque Nationale von Paris erwähnen alle die Heiligen Kosmas und Damian als Urheber des Produktes und bieten mit derjenigen von Basel nur geringfügige Syntaxunterschiede, die Zusammensetzung der Opopira ist die gleiche ${ }^{14}$.

\section{Text der 'Opopira'-Vorschrift in Ms.D III 14, f. 84r, der Universitätsbibliothek Basel}

Sämtliche Kürzungen sind aufgelöst; sie wurden aus drucktechnischen Gründen nicht gekennzeichnet. Die moderne Interpunktion beschränkt sich auf das Setzen von Beistrichen und Strichpunkten zur besseren Trennung der Abschnitte.

Zum Vergleich wurde der Text der Nikolaus-Salernitanus-Bearbeitung nach dem Erstdruck [= Ed.:] des 'Antidotarium Nicolai', Venedig 1471 (kritische Ausgabe von Wouter S.van den Berg [med. Diss.], Leiden 1917; Faksimile bei Goltz [1976] [wie Anm. 1]) herangezogen ${ }^{15}$.

Die zwei Kürzungen, die uns die meiste Mühe verursachten, sind vom Drucker (Herausgeber) des Textes nicht verstanden worden. Es betrifft dies die Buchstaben $\overline{c c}$ in Zeile 20 und das $\overline{0}$ in den Zeilen 22 und 23. Cappelli ${ }^{16}$ gibt weder für $\overline{c c}$ noch für $\overline{\mathbf{o}}$ eine unserer Textübertragung dienende Lösung. Zwei gleiche Buchstaben stehen für eine Pluralbildung, oder sie sind von zwei gleich anlautenden Silben übernommen. In unserem Text trifft letzteres zu: corticis - wir haben es hier mit einer syllabaren Suspension zu tun. Sie kommt verhältnismäßig selten vor. 
Das $\overline{0}$ ist eine Kontraktion, die hier für omnium steht; darauf kommen wir in der Anmerkung bei Zeile 22 zurück.

\section{Text}

1.

2 .

Opopira ma-

3 .

gna, quam sanctissimi viri

Cosmas et Damianus dictaverunt,

4.

guttamque oculumque, labia et linguam

5.

distemperat, ut recte sermonem qui non pro-

6.

ferat aut non loquatur, peculialiter

7. curat, et paralisis gutturis, brachii,

8. manuum, crurum, pedum ac totius

9. corporis optime medetur cum apozimate

10 . salviae, costi atque castorei; contractio-

11 . ni nervorum subvenit; epilempicis,

12. melancolicis, maniacis cum decoctione thuris masculi auxiliatur; tus-

13. sientibus et omnibus doloribus pectoris et stomachi cum decoctione calamentis

14. et cimini; epaticis cum apozimate apii, eupatorii; spleneticis cum de-

15. coctione caparis et absinthii; pleureticis cum apozimate ysopi et

16. pul[e]gii; yliosis cum decoctione yris et macedonici; calculosis cum

17. decoctione saxifragae et ozimi; colicis cum decoctione polipodii

18. et parthenotidos; tipice febrientibus cum apozimate pigani,

19. strucii et pyretri prodest et ad alias multas passiones corporis facit. Recipe opii

20. thebaici dragmas XXXVI, cinamomi, gariofili, nardi, galangae, corticis cos-

21. ti, zedoariae, zinziberis, xilobalsami, reupontici, piretri, corralli, dragaganti, diagridii,

22. mirrae troclitae, castorei, opoponacis, piperis nigri et albi et longi omnium XVIII dragmas XXXVI et semis,

23. rasurae eboris, lilifagi, herbae paralisis omnium III dragmas XVIII, balsami, musci, xi-

24. loaloes, squinanti, cassiae fistulae, storacis, calamentis, masticis, confitae rubeae, gal-

25. bani, serapini, lasaris, melliloti, aristolochiae rotundae, dragunteae, asari,

26. ellebori nigri, meu (mei), pioniae, rafani, corticis peucedani, acori, man- 


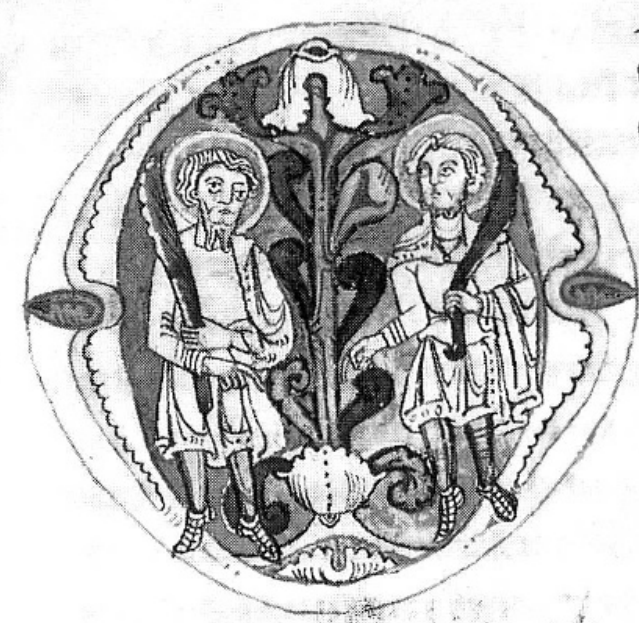

\section{POP 1 BA. OA-}

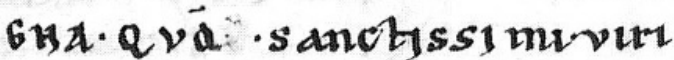

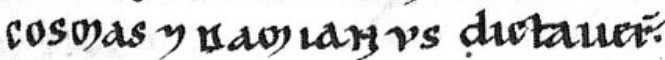
guträq; octm q; Labraylunguam dutenpat.ut rectefermoni ferat au ñloguat pecullalter

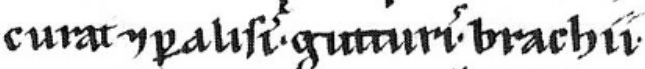

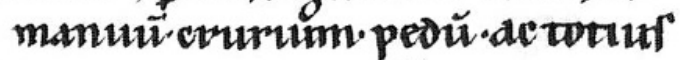
corptr oprin medor. cul aposmatre Calue coftu arq; caltoret comittio nineruos fubuente epilenters.

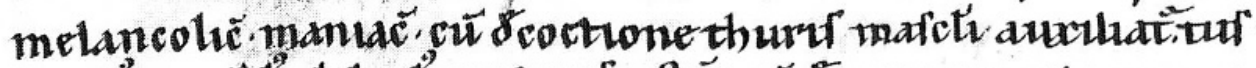

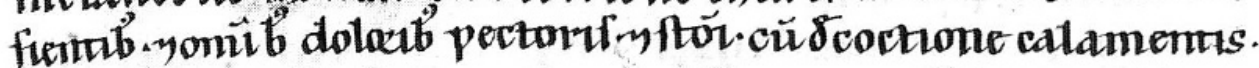

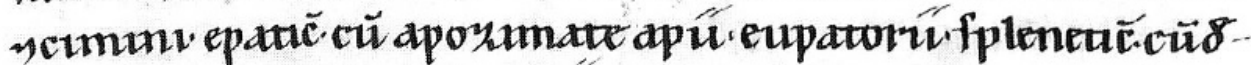

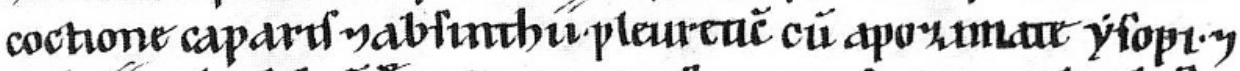

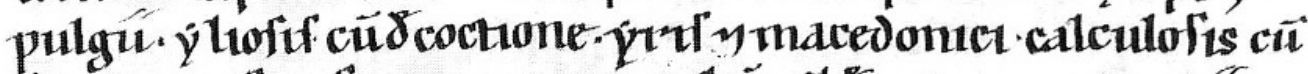
scoctione faxtiage y yumi colič cü dectione polipodí.

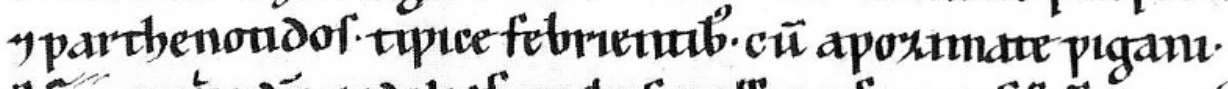

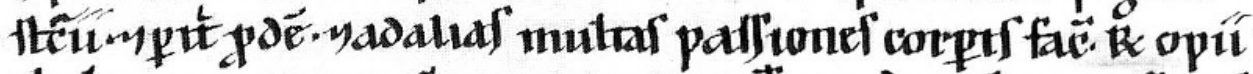

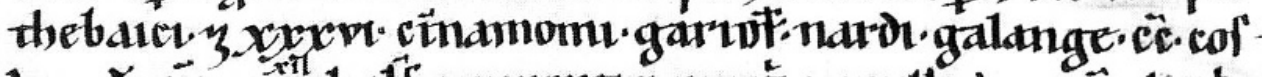

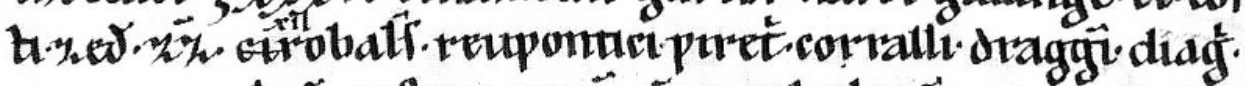

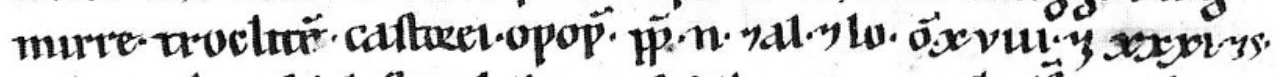

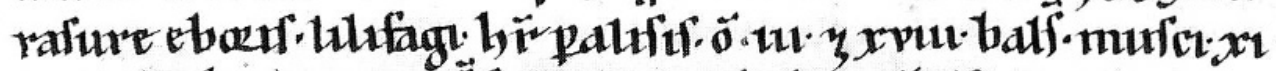

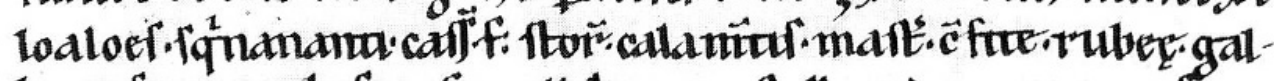

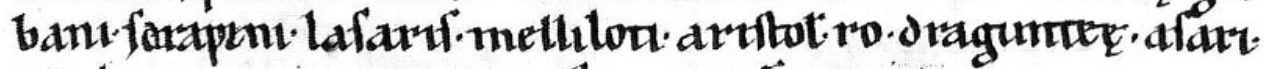
elleboznt meu puonte rafancontric peucedant dcom man -

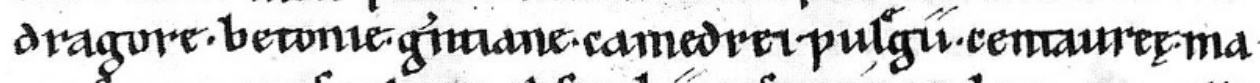

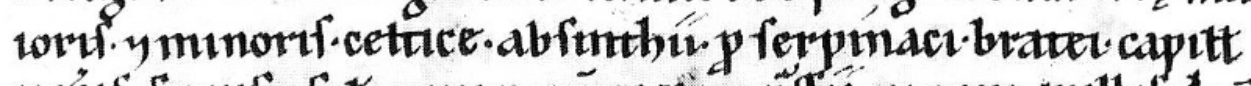

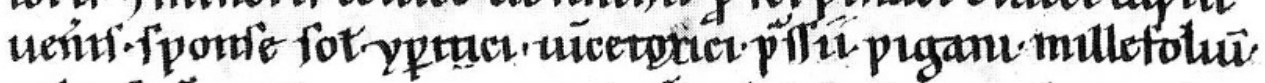

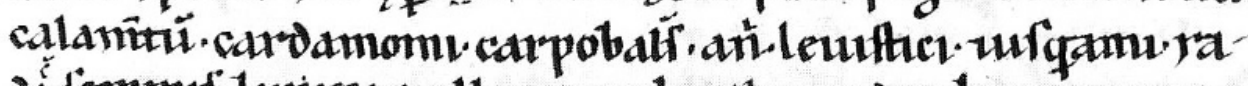

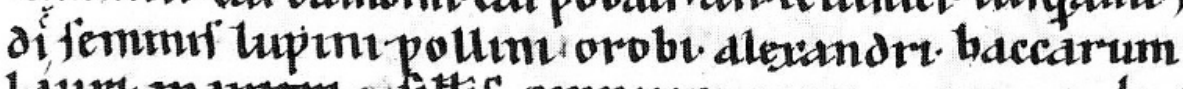

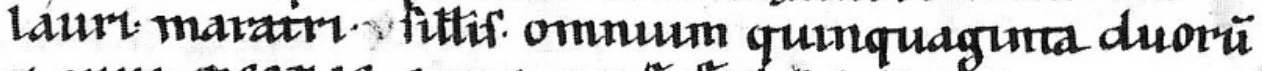

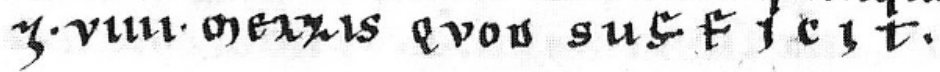


27. dragorae, betoniae, gentianae cam. edrei, pul[e]gii, centaureae ma-

28. ioris et minoris, celtice, absinthii, proserpinaci, brateis, capilli

29. veneris, sponsae solis, yperitici, vincetoxici, prasii, pigani, millefolium,

30. calamentum, cardamomi, carpobalsami, anisi, leuistici, iusquiami ra-

31. dicis, seminis lupini, pollinis orobi, alexandrini, baccarum

32. lauri, maratri et sileris: omnium quinquaginta duorum

33. dragmas VIIII, mellis quod sufficit.

\section{Übersetzung des Textes}

Die große Opopira, welche die hochheiligen Kosmas und Damian haben aufschreiben lassen: das Mittel erstreckt [seine Wirkung] über die Lippen, die Zunge und den grauen Star; ebenso pflegt es besonders denjenigen, der das rechte Wort weder herausbringt noch sprechen kann; und bestens geheilt werden Lähmungen des Kehlkopfes, der Arme, Hände, Schenkel, Füsse und des ganzen Körpers mit einer Abkochung von Salbei, Kost und Bibergeil; bei Kontrakturen von Bändern und Sehnen nützt es; Epileptikern, Melancholikern, manischen Patienten wird mit einem Dekokt von Weihrauch geholfen; bei Husten sowie allen Brust- und Magenschmerzen eine Abkochung von Bergminze und Kümmel; Leberkranken [hilft es] mit der Abkochung von Sellerie, Wildem Salbei; Milzkranken mit Kapern- und Wermutdekokt; Lungenkranken mit Hyssop- und Poleidekokt; Magen-Darm-Leidenden mit einer Abkochung von Schwertlilie und mazedonischer Petersilie; Blasenstein-Patienten mit einem Dekokt von Steinbrech und Basilikum; Kolikleidenden mit Wurmfarn- und Bingelkrautabkochung; bei typicaFieber sowie vielen anderen Körperleiden nützt es mit einer Abkochung von Wilder Raute, Brunnenkresse und Römischem Bertram. Man nehme: Thebais-Opium, Zimt, Gewürznelken, Nardenbaldrian, Galgant, Kostrinde, Zitwer, Ingwer, Balsamholz, Rhapontischen Rhabarber, Römischen Bertram, Korallen, Traganth, Purgierwinde, Runde Myrrhe, Bibergeil, Gummiwurz, Pfeffer (Schwarzen, Weissen und Langen), 361\%2 Drachmen von allen 18; geraspeltes Elfenbein, Grünen (Wilden) Salbei, Himmelschlüssel, 18 Drachmen von allen dreien; Balsam(saft?), Moschus, Riechholz, Kamelgras, Röhrenkassie, Styrax, Bergminze, Mastix, «rotes Konfekt», Galbanum, Serapinum, Teufelsdreck, Honigklee, Runde Osterluzei, Drachenwurz, Haselwurz, Schwarze Nieswurz (Christrose), Bärwurz, Pfingstrose, Rettich, Rinde vom Echten Haarstrang, Kalmus, Alraune, Betonie, 
Enzian, Edelgamander, Polei, Großes und Kleines Tausendgüldenkraut, Speik, Wermut, Vogelknöterich, Sadebaum, Frauenhaar, Schwalbenwurz, Wilde Raute, Schafgarbe, Bergminze, Kardamon, Balsamfrucht, Anis, Liebstöckel, Bilsenkraut (-Wurzel, oder: Bilsenkraut, Rettich), Lupinensamen, Walderbsenmehl, Fenchel, Lorbeeren, Bergfenchel und Roßkümmel, 19 Drachmen von allen 52. Von Honig eine genügende Menge.

\section{Kommentar zum Text (Zeilenzählung)}

1 Der Name wird in der Ed. etymologisiert: opopira dicitur a succo: \& igne. opos enim grece latine succus. pir id est ignis. inde opopira idest ignitus succus.

2/3 Der Hinweis auf Kosmas und Damian fehlt in der Ed.

$4 \quad$ Ed.: linguam \& labiam

$6 \quad$ Ed.: peculiariter

$7 \quad$ Ed. folgt: oris \& gutturis; brachii fehlt

8/9 Ed.: \& pedum atque crurum \& totius corporis

9 Ed. folgt nach cum: uino ubi cocta fit saluia

10 Ed. hat hier eine Indikation, Zubereitung und Dosierungsangabe mehr

11 Ed. folgt nach subvenit: colericis cum decoctione polipodii (s. Zeile $17 / 18)$

12 Ed.: multis [statt melancolicis] et maniacis

13 Ed.: in omnibus

17 Ed.: apozimate saxifragiae

17/18 Ed. fehlt: colicis cum decoctione polipodii et parthenotidos (s. Zeile 11)

18 Ed.: decoctione pigami für apozimate pigani

19 Ed.: nasturcii für strucii

20 Ed.: Der Herausgeber (Drucker) hat mit der Abbreviatur $\overline{c c}$ nichts anzufangen gewusst und sie deshalb so stehen gelassen!

21 Ed.: coralli albi et rubri; fehlt: diagridii

22 Ed.: troclitae fehlt

Die ungebräuliche Abbreviatur $\bar{o}$ für omnium wurde nicht verstanden. Der Herausgeber hat sich um das Problem gedrückt und die Stelle mit ana zu lösen versucht. Omnium xviii. «von allen 18» stimmt jedoch, wenn man die vorher genannten Drogen auszählt. Dabei sind dann piper nigrum, album et longum als eine Droge aufzufassen. Obendrein ergibt sich dadurch die Lösung für $\overline{\mathrm{cc}}$, womit nur corticis - 
gut passend zu costi-und nicht eine weitere Droge gemeint sein kann.

23 Entsprechend ist hier $\overline{\mathbf{o}}$ als omnium zu lesen. Die Bestätigung findet sich übrigens noch in Zeile 32. Merkwürdigerweise hat der Erstdruck dort auch ana, was für die Bestimmung der Vorlage von Bedeutung sein kann.

25 Ed.: asari [!]; in der gleichen Zeile aber auch assari, so daß das erste asari wohl eine fehlerhafte Lesung für lasaris ist.

27 Ed.: betonice

28 Ed.: blactei für brateis

$30 \quad$ Ed.: seminis anisi

30/31 Es fällt auf, daß in der Ed. radicis fehlt. Gehört radicis vielleicht zu iusquiami? Möglich ist es.

\section{Kommentar zur Arzneimittelgeschichte (Zeilenzählung)}

Zur Arzneimittelgeschichte gibt es eine umfangreiche Literatur ${ }^{17}$. Deshalb beschränken wir uns auf die notwendigsten Erläuterungen.

5 distemperat: Terminus der Humoraltherapie. Die gestörte Säftemischung (dyskrasis) wird ins Gleichgewicht (eukrasis) gebracht, wird temperiert.

9 apozima: apozema > gr. zein, (sieden). ${ }^{18}$

12 thus masculinum: «Est optimum quod masculum dicitur, subrufum». ${ }^{19}$ Roter Weihrauch, von Boswellia sacra Flückiger.

16 macedonicum: Für Petersilien-Arten finden sich in der antiken und mittelalterlichen Literatur die Bezeichnungen macedonicum, alexandrinum uel oxilatrum ${ }^{20}$.

17 polipodium: Pteris; wsch. Dryopteris filix-mas (L.) Schott ${ }^{21}$.

18 tipice febrientibus: «febris typica» war eine der in der antiken Medizin differenzierten Fieberarten ${ }^{22}$.

20 nardus: spica nardi, von Nardostachys jatamansi DC.

21 xilobalsamum: Balsamon, mit opobalsamon $=\operatorname{der}$ Saft, xylobalsamon = das Holz und carpobalsamon = die Frucht von Amyris balsamifera L., Balsamstrauch ${ }^{23}$.

21 reuponticum: «simili est reubarbario sed non tingit sicut reubarbarum». ${ }^{24}$ Rhapontischer Rhabarber, Rheum rhaponticum L.

21 diagridium: «scamonia idem», ${ }^{25}$ von Convolvulus scammonia L., Purgierwinde. 
22 mirra troclita: «mira trodiden, ad laudem dicta est quod sit troditen, id est rotunda». ${ }^{26}$ Dazu aber auch: der «Troglodytische Myrrhe» im Text des Dioskurides ${ }^{27}$. Gummiharz von Commiphora-Arten.

22 opopanax: Panakos ${ }^{28}$. Aber auch «opopanax succus est herba que panax dicitur, similis per omnia ferule». ${ }^{29}$ Aus Opopanax chironum (L.) W. D. J. Koch, Gummiwurz. Alter Name: Ferula opopanax Spr.

23 rasura eboris: Abschabsel von Elefantenzähnen, Elfenbein ${ }^{30}$.

23/24 xiloaloes: siloaloes, lignum aloes, Paradiesholz, usw., hat nichts mit Aloe zu tun. Es ist das wohlriechende Kernholz von Aquilaria agallocha Roxb. ${ }^{31}$

24 squinantum: schoenanthum (auch iuncus odoratus). Das echte squinantum stammte vom Riechgras Cymbopogon schoenanthus $L$.

24 confita rubea: Dasjenige, was beim Auspressen der Rinde von Styrax officinalis L. als Styrax, Storax übrigblieb, wurde u. a. confita rubea genannt ${ }^{32}$.

25 serapinum: «gumi cuiusdem arboris est in Grecia». ${ }^{33}$ Serapinum ist wohl eine verderbte Schreibart für sagapenum und dies ist das Gummi einer Ferula-Art.

25 lasar: laser, lacryma laseris, usw. ${ }^{34}$. Eine Art Asa foetida.

26 meu: von Meum athamanticum Jacq., Bärwurz; vielleicht aber von Peucedanum carvifolia Vill., Haarstrang.

27 centaurea maior et minor: Es wurden in der antiken Medizin unterschieden das Große Kentaurion, die Asterazee, Centaurea centaurium L. (und andere C.-Arten), und das Kleine Kentaurion oder Tausendgüldenkraut, die Gentianazee Centaurium minus Moench (C.erythraea Raf.). ${ }^{35}$

28 celtica: von Valeriana celtica L., Echter Speik.

28 proserpinacum: Polygonum aviculare L., Vogelknöterich ${ }^{36}$.

28 brateis (und blacteis der Ed.): Synonyme für Juniperus sabina L., Sadebaum ${ }^{37}$.

29 sponsa solis: Synonym für verschiedene Asterazeen, vielfach aber für Cichorium intybus L., Cichorie, Wegwarte ${ }^{38}$.

29 prassium: Synonym für Andorn, Marrubium vulgare L. ${ }^{39}$

30 carpobalsamum: s. Anm. zu Zeile 21.

31 alexandrinum: s. Anm. zu Zeile 16.

32 maratrum: Es wurden verschiedene Fenchel-Arten - wilde und angebaute - verwendet. Nach Dioskurides: Marathron, war wohl Seseli caespitosum Sibth. et Sm., Bergfenchel ${ }^{40}$. 

siler L., gewesen $\operatorname{sein}^{41}$.

\section{Anmerkungen}

1 Vgl. hierzu A.Lutz: Der verschollene frühsalernitanische Antidotarius magnus in einer Basler Handschrift aus dem 12.Jahrhundert und das Antidotarium Nicolai. Veröff. d.Int.Ges.f.Gesch. d.Pharm., NF, Bd.16, Stuttgart 1960, S.97-133. Im allgemeinen über das Große Antidotar, vgl.etwa W.Braekman/G. Keil, Fünf mittelniederdeutsche Übersetzungen des 'Antidotarium Nicolai', Sudhoffs Arch.55 (1977), S.257-320, D.Goltz: Mittelalterliche Pharmazie und Medizin. Dargestellt an der Geschichte und Inhalt des Antidotarium Nicolai. Veröff.d.Int.Ges.f.d.Pharm., NF, Bd.44, Stuttgart 1976, und W.F. Daems: Alte Pharmazie und Medizin, Ausstellung anläßlich des Int. Kongr.f.Gesch.d.Pharm., Basel, 1979. Arlesheim 1979. Schon Sigerist, aber auch Häfliger, hatten unter anderen Autoren das Große Antidotar erwähnt; vgl. H. Sigerist, Studien zur frühmittelalterlichen Rezeptliteratur, Leipzig 1923, und J.A. Häfliger. Das Apothekenwesen Basels, Basler Zschr.f. Gesch.u. Altertumsk., 36-37 (1937-1938), S. 32.

2 Zuerst von Häfliger; vgl. J.A.Häfliger, a.a.O. Ferner vgl. A.Lutz, a.a.O., ders.: Initialen aus dem «Liber Antidotarius magnus». Basel 1979, sowie W.H.Hein und D. A. Wittop Koning: Die Apotheke in der Buchmalerei. Frankfurt 1981, S. 16-17.

3 Die Initiale wurde schon von Escher beschrieben; vgl. K. Escher: Die Miniaturen in den Basler Bibliotheken, Museen und Archiven, Basel 1917, S.44-45. Sie wurde dann im Rahmen einer Forschungsarbeit über den Kult und die Ikonographie der Heiligen Kosmas und Damian in der Schweiz vor kurzem wieder erwähnt; vgl. hierzu F. Ledermann: Les saints Côme et Damien en Suisse, un aperçu. Schweiz. Apoth. Ztg., 119 (1981), S.592-594 a, und ders.: Les saints Côme et Damian et leurs médicaments: l'opopira dans l'Antidotarius magnus de la Bibliothèque de Bâle. Erschienen in den Akten des Int. Kongr. f. Gesch. d. Pharm. Granada 1985, S.407-410.

4. Über die Quellen, vgl. A. Wittmann: Kosmas und Damian, Kultausbreitung und Volksdevotion. Berlin 1967; P. Julien: Saint Côme et saint Damien, patrons des médecins, chirurgiens et pharmaciens. Paris 1980; F. Ledermann: Les saints Côme et Damien et leurs médicaments, a.a.O.

5 Vgl.hierzu P.Julien, a.a.O. Ferner zum remedium caeleste, vgl. R.Schmitz: Mörser, Kolben und Phiolen. 2. Aufl. Graz 1978, S.51.

6 Zur Gesamt-Thematik der diversen Devotionswellen, vgl. M.L. David-Danel: Iconographie des saints médecins Côme et Damien. Lille 1958; P. Julien: Côme et Damien hier et aujourd'hui. Quelques questions. In P. Julien und F. Ledermann: Die Heiligen Kosmas und Damian, Kult und Ikonographie. Veröff.d.Schweiz.Ges.f.Gesch.d.Pharm., Bd.5, Zürich 1985, S.43-63.

7 Vgl. P. Julien: Saint Côme et saint Damien, a. a. O. Ferner vgl. F. Ledermann: Les saints Côme et Damien et leurs médicaments, a.a.O.

8 Constantinus Africanus: Opera. Basilea 1536, Bd.I, S.207. 
9 Vgl. etwa D.Goltz, a. a.O., sowie G. Keil in: Lexikon des Mittelalters, I, München/Zürich 1977-1980, Sp. 708-710.

10 Vgl. W.F.Daems, a.a. O., sowie G. Keil, a.a.O.

11 Vgl. Arnaldus Villanova: Opera omnia, Basel 1585, S. 455.

12 Vgl. hierzu W.Schneider: Lexikon zur Arzneimittelgeschichte. Frankfurt am Main 19681975.

13 D. III 14, f. $84 \mathrm{r}$.

14 Ms. Lat. 7009, Ms. Lat. 7010, Ms. Lat.16187.

15 D.Goltz, a.a.O., liefert eine Fak-Simile Ausgabe dieser Fassung des «Antidotarium Nicolai».

16 Vgl. A.Cappelli: Dizionario di abbreviature latine ed italiane. Milano 1949.

17 Vgl. etwa W. Schneider, a.a. O.

18 Vgl. Lemma 'Arzneiformen' (W.F.Daems), in: Lexikon des Mittelalters I(6) München/Zürich (1980), Sp. 1094-1096.

19 Vgl. J.L.G. Mowat: A medico-botanical glossary from the Bodleian Manuscript. Selden B. 35. Oxford 1887 (= Alphita), S. 188.

20 Ebda., S.139 und 5. Ferner, vgl. W.F.Daems: Nomina simplicium medicinarum ex synonymariis medii aevi collecta. In Vorbereitung (1988). Lemmata 365, 633.

21 J.Berendes: Des Pedanios Dioskurides aus Anazarbos Arzneimittellehre in fünf Büchern. Stuttgart 1902 (Nachdruck: Wiesbaden 1970), S.472: Diosk. IV, 183.

22 Vgl. W. Anschütz, Zwei Fieberschriften des Breslauer Codex Salernitanus, med. Diss. Leipzig 1919.

23 Vgl. J.Berendes, a. a. O.: Diosk. I. 18.

24 Vgl. J.L. Mowat, a. a. O., S. 157.

25 Ebda., S.50.

26 Ebda., S.119.

27 Vgl. J.Berendes, a.a.0., S. 80: Diosk.I.77.

28 Ebda.: Diosk. III. 48.

29 Vgl. J.L.G. Mowat, a. a.O., S.130.

30 Vgl. W.Schneider, a. a.O., Bd.I, S. 35.

31 Ebda., Bd. V/1, S.112.

32 Vgl. W(outer) S. van den Berg: Eene Middelnederlandsche vertaling van het Antidotarium Nicolaï. Leiden 1917, S.228 und 268.

33 Vgl. J.L.G.Mowat, a.a.O., S.166.

34 Vgl. W.Schneider, a.a.O., Bd. V/2, S.93.

35 Vgl. W.F. Daems: Nomina ..., a. a. O., 64, 111, 212, 213, 591.

36 Ebda., 384.

37 Ebda., 101, 418.

38 Ebda., 161, 199, 432, 565, 578. Ferner vgl. W.F. Daems: Terminologische Probleme mittelalterlicher Pharmakobotanik. Ber. Physico-Medica (Würzburg) 88 (1981/1983), S. 97-109. Würzburg 1984, hier: S.107.

39 Vgl. W.F. Daems: Nomina..., a. a. O., 305, 377, 648, 678.

40 Vgl. J. Berendes, a. a. O.: Diosk. III, 74.

41 Vgl. W.Schneider, a. a. O., Bd.V/2, S.232. 


\section{Zusammenfassung}

Eine Initiale der Handschrift des Antidotarius magnus der Universitätsbibliothek Basel zeigt die Heiligen Kosmas und Damian, welche eine pharmazeutische Vorschrift begleiten, die große Opopira. Die Autoren liefern eine kommentierte Edition sowie eine Übersetzung des Textes und zeigen, daß der Ursprung dieser pharmazeutischen Zusammensetzung in der salernitanischen Tradition zu suchen ist.

\section{Summary}

The 'Antidotarius magnus' Manuscript of the University Library, Basle, depicts in the initial $\mathrm{O}$ of the prescription for 'Opopira magna' the saints Cosmas and Damian. The authors furnish a critical edition and translation of the text and show the prescription to be of salernitan origin.

\section{Résumé}

Sur le manuscrit de l'Antidotarius magnus de la Bibliothèque universitaire de Bâle, une miniature historiée présente les saints Côme et Damien accompagnant une formule pharmaceutique: le grand opopira. Les auteurs fournissent une édition annotée ainsi qu'une traduction de ce texte et montrent que cette composition pharmaceutique est d'origine salernitaine.

Dr. Willem F. Daems

Weleda AG

Stollenrain 15

CH-4144 Arlesheim

Dr. François Ledermann

Schloß-Apotheke

Könizstraße 3

CH-3000 Bern 21 